\title{
Optimization of WSNs Flooding Rates by Khalimsky Topology
}

\author{
${ }^{1}$ Mahmoud Mezghani, ${ }^{2}$ Riyadh Gargouri and ${ }^{3}$ Mahmoud Abdellaoui \\ ${ }^{1}$ National Engineering School of Sfax (ENIS), Sfax University, Tunisia; \\ ${ }^{2}$ Department of Sciences Taibah University, Saudi Arabia; \\ ${ }^{3}$ Engineering School of Electronics and Communication of Sfax (ENET'COM), Sfax University, Tunisia; \\ ${ }^{1}$ mahmoud.mezghani@gmail.com; ${ }^{2}$ tn_riadh_30@yahoo.fr; ${ }^{3}$ mahmoudabdellaoui4@gmail.com
}

\begin{abstract}
In this paper, we proposed a new method of deploying and building an organized architecture of gateway nodes in a Wireless Sensors Network (WSN) formed also by randomly deployed sensors arranged in clusters. This method, based on the Khalimsky theory, reduces the energy consumption and the flooding rates of the conventional flooding algorithm.

Our solution allowed to build a hierarchical topology reduces the number of communication links between nodes while maintaining the adjacency list to minimize the data redundancy.

It allows calculating an optimal set of forwarding gateway nodes to route data packets between a source and a destination. This set includes all optimal paths using the less number of nodes. Our simulation study shows that the Khalimsky theory reduces considerably the flooding rates and the energy consumption.

Our solution can be applied in the fields needing prefixed gateway nodes and randomly deployed sensors which use urgent data with real-time aspect such as the security and the monitoring of industrial zones and home building.
\end{abstract}

Keywords: Wireless Sensor Network; WSN; Flooding; Khalimsky Topology; Flooding Khalimsky Algorithm; Flooding Rates; Energy Consumption; WSN Deployment.

\section{Introduction}

Technological advances in recent years have enabled the development of new types of sensors equipped with wireless communication system that can be configured to form autonomous networks called WSNs. These WSNs are used in various domains such as home automation, health care, military domain and environment monitoring. In the network, the sensors nodes cooperate between them to treat and to convey the collected data towards the base station which acts according to programmed scenarios. These sensors are characterized by a small memory size, a low capacity for treatment and limited energy resource [1, 2].

Generally, dissipated energy by a sensor is the total of energies used to gather, treat and send/receive data. Most of this energy is used by the radio module in sending/receiving information. The main focus of WSN research has been on energy efficiency. The objective is the development of topologies and routing protocols allowing the reduction of the dissipated energy by the radio component. We should note that the effectiveness of a routing protocol depends on the architecture of sensors nodes deployment and their operating processes. For that, flooding is very used for the WSN architecture construction. It identifies relationships between nodes and their 
Mahmoud Mezghani, Riyadh Gargouri and Mahmoud Abdellaoui; Optimization of WSNs Flooding Rates by Khalimsky Topology, Transactions on Networks and Communications, Volume 2 No 6, Dec (2014); pp: 25-38

positions to calculate and maintain the routing tables. It consumes a lot of energy and needs to be refined and optimized to eliminate unnecessary data redundancy $[3,4]$.

Accordingly, we devote our research tasks to adapt scientific advances research in the fields of mathematics such as Khalimsky theory and the multi-agents systems to carry out a sensors deployment method for WSNs reducing the flooding rates and minimizing the energy consumption. Our idea consists to create a WSN formed by a set of fixed sensors used as gateway nodes based on the Khalimsky topology and a set of clusters containing member nodes to gather information. The Khalimsky topology permits to convey the flooding process using optimal paths between gateway nodes and the sink when a node transmits a data packet. The multi agents system permits to assign the jobs for each node in the clusters and in the set of the gateway nodes to complete a task of collecting and transmitting information between a source and a destination. Our objective is to minimize the flooding rates by the limiting of the flooding in the set of the gateways, based on the Khalimsky architecture, using only those which form optimal paths.

In this paper, we present Khalimsky topology used to build the architecture of deployed gateways in a WSN. This topology provides a hierarchical architecture in which each sensor node is capable to convey data to their destination in an optimal set of nodes minimizing the flooding rates. In the second time, we present the evaluation results of this method compared with conventional flooding algorithm. This evaluation is carried out by the TOSSIM simulator and the PowerTOSSIM-Z module which allows to model power consumption by sensors nodes using the TinyOS2.1 operating system [5-7].

The remainder of this paper is organized as follows: section 2 presents an outline on WSN deployment architectures, types of routing protocols and flooding problems such energy consumption and information redundancies. Section 3 shows the Khalimsky topology characteristics and the auto-organization algorithm of sensor nodes. Section 4 shows the novel flooding algorithm according to Khalimsky theory. Section 5 presents the simulation and evaluation results of the developed algorithms. Section 6 presents some future works. Finally, section 7 gives some conclusions.

\section{Related Work}

Several research studies have appeared to optimize the energy consumption of nodes through the use of innovative conservation methods to improve network performance, including the maximization of its life. Since the radio module is the greediest component, the trend was to reduce the communications rates by optimizing the data routing to reduce the energy consumption. Consequently, several routing protocols have been developed. However, they cannot be applied appropriately to any application context. Depending on the architecture, the deployed sensors take place by a random or organized way. In the organized way, sensors must be deployed in predetermined positions where it is possible to program their activities and data packets routes. In the random way, the nodes are scattered on the collecting field in mass by several means such as throwing them by plane. In this mode, an auto-organization algorithm is necessary to improve the performance of the WSN [8].

In the literature, WSNs routing protocols can be classified in three main classes: flat, hierarchical and geographic network routing protocols.

In flat architecture, each node plays the same role and has the same functionality as other sensor nodes in the network. Sensors can send data to the base station directly using a high power, or hop 
by hop with a very low power. The most popular flat-based routing protocol is data-centric protocols like flooding. In this type of protocols, it is not necessary to have an addressing mechanism for sensor nodes. Data is propagated gradually to the interested neighbors requiring the data announced or in flood towards selected or all the neighbors with an important redundancy until reaching the base station [8-10]. The unnecessary flooded data packets, the not optimum paths, the data transmission time and the different ratios of energy consumption between sensor nodes are the most disadvantages in the dense flat networks.

In hierarchical architecture, the node representing the cluster-head transmits directly collected data from member-nodes to the base station, or via a multi-hop mode between the cluster-heads. The cluster-heads are generally a sensors more powerful than the member nodes or sensors whose battery level is the highest in the cluster. This type of protocol is the most adopted today. It minimizes more the energy consumption compared to flat architecture and it has two great advantages: scalability and the simple implementation of the aggregation mechanisms. Leach, TEEN and PEGASIS are the most popular hierarchical routing protocols for WSNs [8-10]. The data propagation between the cluster-heads and the sink uses multiple paths which are not necessary optimized. It requires an auto-organization algorithm to select cluster heads and to affect member nodes to the calculated cluster.

In a geographic routing protocol, a node is supposed to know its geographical position, its neighbors and the destination node. To transmit data to the sink, the nearest node destination, among the neighboring nodes, is selected as the next hop. Multiple geographic routing protocols like GPSR, SPEED, GEAR, GAF ... are proposed. In such protocol, the routing data is optimized and the cost to control the algorithm is reduced. However, the disadvantage of geographic-based routing protocols is the means used for nodes localization. The localization is ensured by the exchange of a big number of messages by flooding algorithm to discover nodes between them [8-10].

The flooding mechanism is used by several routing protocols for sensor nodes organization. It permits the routes setup, the neighboring discovery for each node and the links state updates. Such mechanism suffers from a huge amount of information redundancy due to the big number of exchanged auto-organization data packets. However, the reduction of the flooding rates is imperative in order to reduce data redundancy and to minimize energy consumption. For all these reasons, we devoted this paper to the communications energy efficiency in WSNs. The main objective of our work consists to develop a WSN auto-organization method optimizing the communication links between sensors and reducing the flooding rates. For that, we used the mathematical Khalimsky theory to construct a geographic-hierarchical topology [11-15]. This theory permits to calculate the optimized paths when a sensor need to forward data. Theses paths are formed by a succession of nodes belonging to an employed optimal set of gateways between a source and destination.

\section{WSN Deployment according to Khalimsky Topology}

In this section we present the Khalimsky theory and the developed algorithms for the WSN deployment and the flooding of data packets between the sensors and the sink.

\subsection{Khalimsky theory definitions}

In what follows, we present some terminology and definitions necessary for understanding the results of the Khalimsky topology. 
Let $\mathcal{B}=\{\{2 n+1\} ; n \in \mathbb{Z}\} \cup\{\{2 n-1,2 n, 2 n+1\} ; n \in \mathbb{Z}\}$ be a family of subsets of the set of integers $\mathbb{Z}$. Then $\mathcal{B}$ is a base of a topology on $\mathbb{Z}$ called the Khalimsky topology and denoted by $K$. The Khalimsky plane is the Cartesian product of two Khalimsky topologies $(\mathbb{Z}, K) \times(\mathbb{Z}, K)$. This topology on $\mathbb{Z}^{2}$ is characterized as follows. A point at its two coordinates of the form $\left(2 k ; 2 k^{\prime}\right)$ is closed point (they are shown in figure 1 by the black squares), if both coordinates are of the form $\left(2 k+1 ; 2 k^{\prime}+\right.$ 1) point is an open point (shown in the figure 1 by the white squares). These types of points are called "pure points". Other points are called "mixed points" (presented by two lines). It is clearly that $\left(\mathbb{Z}^{2}, k\right)$ is connected space [11-15]. Figure 1 show a connectivity graph of the Khalimsky topology.

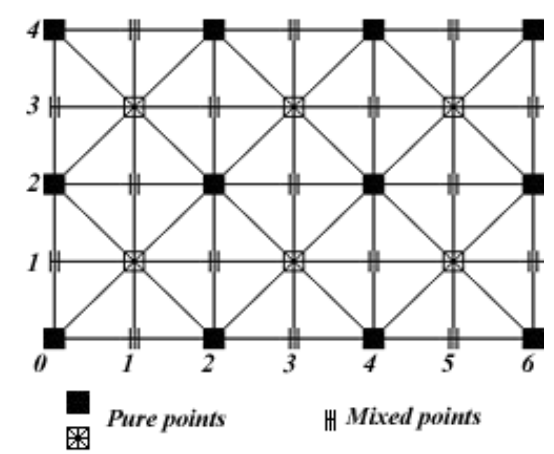

Figure 1: Connexity graph part of Khalimsky topology on $\mathbb{Z}^{2}$

According to the Khalimsky topology, we define:

o $S_{\left(X_{S}, Y_{S}\right)}$ : Source point.

o $Q_{\left(X_{Q}, Y_{Q}\right)}$ : Destination point.

o $A_{S}^{Q}$ : A digital arc joining the points $S$ and $Q$.

o $\quad M_{S}^{Q}$ : A minimal digital arc joining the points $S$ and $Q$.

o $\quad B L(S, Q)=\cup M_{S}^{Q}$ : The whole of minimal digital arcs joining the points $S$ and $Q$.

o $d_{\infty}(S, Q)=\max \left(\left|X_{Q}-X_{S}\right|,\left|Y_{Q}-Y_{S}\right|\right):$ The norm in $\mathbb{Z}^{2}$

According to these definitions, we define:

o $S_{\left(X_{S}, Y_{S}\right)}$ : Source sensor node.

o $Q_{\left(X_{Q}, Y_{Q}\right)}$ : Destination sensor node.

o $\quad A_{S}^{Q}$ : A link regrouping a set of intermediate sensors joining two sensor nodes $S$ and $Q$.

o $M_{S}^{Q}:$ A minimal link joining the sensor nodes $S$ and $Q$.

o $\quad B L(S, Q)=\cup M_{S}^{Q}$ : The whole of minimal link joining the sensor nodes $S$ and $Q$.

o $d_{\infty}(S, Q)$ : The number of sensors in the link joining nodes $S$ and $Q$.

\subsection{Khalimsky deployment algorithm}

According to the previous definitions, our algorithm permits the deployment of the gateway sensors in the collecting field as the Khalimsky topology as shown in figure 2. The gateway nodes in this topology are either pure or mix. Pure nodes have 8 neighbors and mix nodes have only 4 neighbors. Mix nodes communicate with pure nodes only. The nodes are organized in levels. The $\operatorname{sink} S_{(0,0)}$ belongs to the level 0 its neighbors belong to the level 1 and respectively until level $n$. 


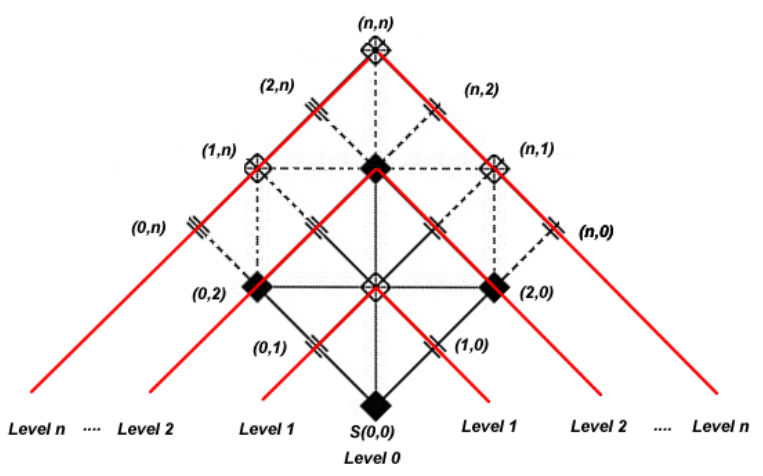

Figure 2: Sensors coordinates assignment in the Khalimsky topology

To create the topology, the sink $\mathrm{S}_{(0,0)}$ diffuses a message towards all the nodes in its range to allow the coordinates between them. This message is a structure composed by five variables:

O IDSender : the identifier of the sender node,

o (XSender, YSender): the coordinates of the sender node,

o NISender : the level of the sender in the Khalimsky hierarchy,

o NB: the nodes number in the current level of the sender node.

The NB variable is calculated by the following $\left(U_{n}\right)$ :

$$
U_{n}=\left\{\begin{array}{c}
U_{0}=1 \\
U_{n+1}=U_{n}+2
\end{array}\right.
$$

Each node receiving the message, run the auto-organization algorithm as shown in the following algorithm to calculate its coordinates of the auto-organization structure, and then diffuses them towards the nodes in its range.

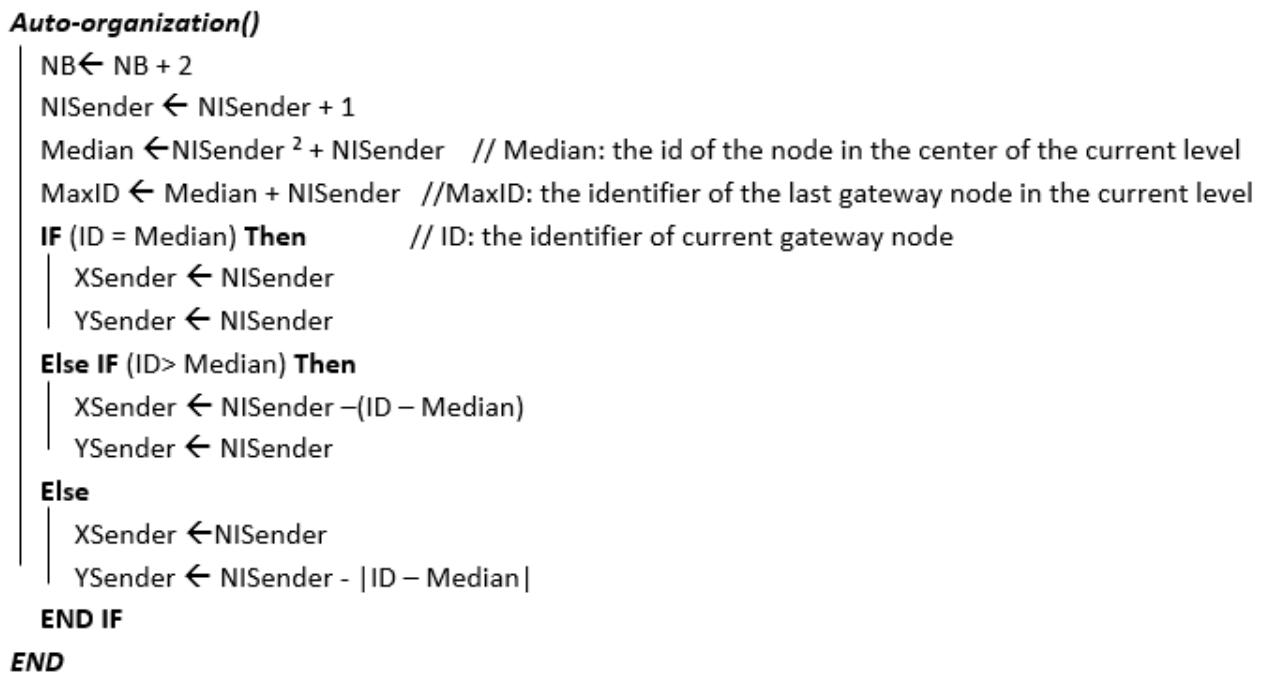

Algorithm 1: Gateway sensor nodes auto-organization algorithm

\section{Khalimsky Flooding Algorithm}

In order to avoid data redundancy and the problems due to the implosion and the overlapping caused by the conventional flooding approach, we developed a novel algorithm based on the Khalimsky topology which reduces the field of used nodes when a source sensor $\mathrm{Q}_{\left(\mathrm{X}_{\mathrm{Q}}, \mathrm{Y}_{\mathrm{Q}}\right)}$ broadcasts data to the sink $S_{(0,0)}$. This field is defined by $B L(S, Q)=U M_{S}^{Q}$ the set of nodes forming all minimal links which connect the source node $Q_{\left(X_{Q}, Y_{Q}\right)}$ to the sink $S_{(0,0)}$. Figure 3 shows $B L(S, Q)$ of a nodes set used when a sensor $Q_{\left(X_{Q}, Y_{Q}\right)}$ broadcasts data to the $\operatorname{sink} S_{(0,0)}$. 


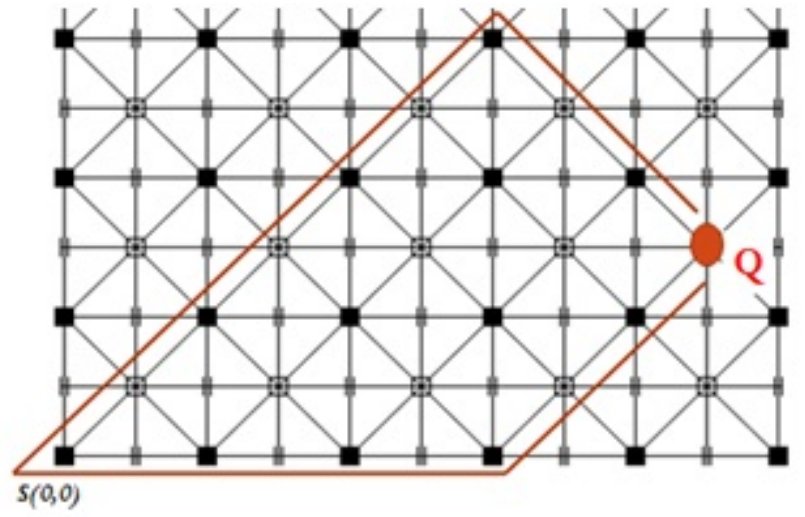

Figure 3: $\mathrm{BL}(\mathrm{S}, \mathbf{Q})$ of nodes belonging minimal paths between $\mathbf{Q}_{\left(\mathrm{x}_{\mathbf{Q}}, \mathrm{Y}_{\mathbf{Q}}\right)}$ and $\mathbf{S}_{(0,0)}$

\section{1 $B L(S, Q)$ Construction}

Khalimsky topology distinguishes five cases to determine the lists of nodes to traverse to form the minimal paths separating two nodes $\mathrm{Q}_{\left(\mathrm{X}_{\mathrm{Q}}, \mathrm{Y}_{\mathrm{Q}}\right)}$ and $\mathrm{S}_{(0,0)}$ [11-15]. These nodes are in the zone I; II; III or IV; as well as, on the lines $\mathrm{D}_{(\mathrm{Y}=\mathrm{X})}$ or $\mathrm{D}_{(\mathrm{Y}=-\mathrm{X})}$ as shown in figure 4.

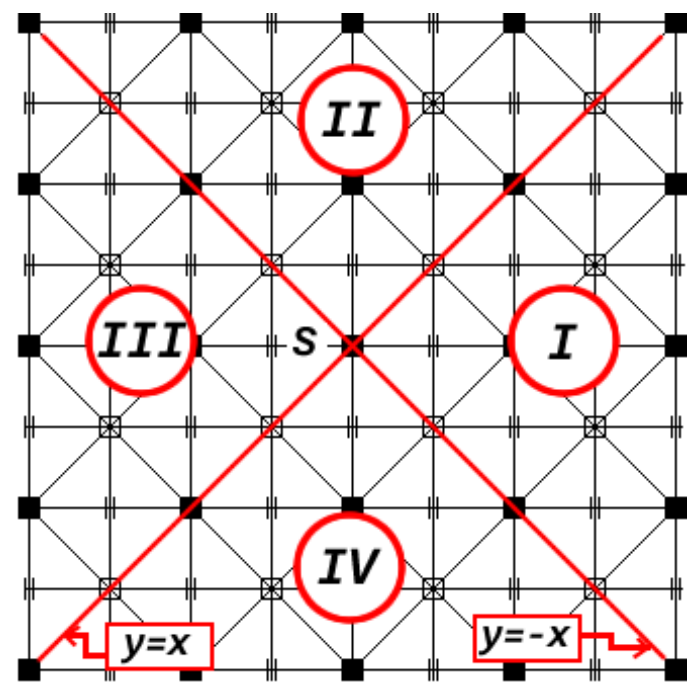

Figure 4: Minimal path zones in Khalimsky topology on $\mathbb{Z}^{2}$

In the following, P represents an intermediate sensor node in the optimal whole of sensors joining two nodes $S_{\left(X_{S}, Y_{S}\right)}$ and $\mathrm{Q}_{\left(\mathrm{X}_{\mathrm{Q}}, \mathrm{Y}_{\mathrm{Q}}\right)}$.

0 Case 1 : If $S$ is a pure node and $\left|X_{Q}-X_{S}\right|=\left|Y_{Q}-Y_{S}\right|$ then $Q$ is a pure node and

$$
\mathrm{P} \in \mathrm{BL}(\mathrm{S}, \mathrm{Q}) \Leftrightarrow\left\{\begin{array}{c}
\left|\mathrm{X}_{\mathrm{P}}-\mathrm{X}_{\mathrm{S}}\right|+\left|\mathrm{X}_{\mathrm{Q}}-\mathrm{X}_{\mathrm{p}}\right|=\left|\mathrm{X}_{\mathrm{Q}}-\mathrm{X}_{\mathrm{S}}\right| \\
\left|\mathrm{Y}_{\mathrm{P}}-\mathrm{Y}_{\mathrm{S}}\right|+\left|\mathrm{Y}_{\mathrm{Q}}-\mathrm{Y}_{\mathrm{p}}\right|=\left|\mathrm{Y}_{\mathrm{Q}}-\mathrm{Y}_{\mathrm{S}}\right|
\end{array}\right.
$$

The set of sensors joining nodes $\mathrm{S}_{(0,0)}$ and $\mathrm{Q}_{\left(\mathrm{X}_{\mathrm{Q}}, \mathrm{Y}_{\mathrm{Q}}\right)}$ are on the lines $\mathrm{D}_{(\mathrm{Y}=\mathrm{X})}$ or $\mathrm{D}_{(\mathrm{Y}=-\mathrm{X})}$.

0 Case 2: If $S$ is a mixed node and $\left|X_{Q}-X_{S}\right|=\left|Y_{Q}-Y_{S}\right|$ then $Q$ is a mixed node and

$$
\begin{array}{r}
P \in B L(S, Q) \Leftrightarrow \operatorname{BL}(S, Q)=B L\left(S_{1}, Q_{1}\right) \cup B L\left(S_{2}, Q_{2}\right) \cup\{S, Q\} \\
\text { With } \quad S_{1}\left(X_{S}, Y_{S}-\frac{Y_{S}-Y_{Q}}{\left|Y_{S}-Y_{Q}\right|}\right), S_{2}\left(X_{S}-\frac{X_{S}-X_{Q}}{\left|X_{S}-X_{Q}\right|}, Y_{S}\right), \\
Q_{1}\left(X_{Q}-\frac{X_{Q}-X_{S}}{\left|X_{Q}-X_{S}\right|}, Y_{Q}\right), Q_{2}\left(X_{Q}, Y_{Q}-\frac{Y_{Q}-Y_{S}}{\left|Y_{S}-Y_{Q}\right|}\right)
\end{array}
$$


When $S_{\left(X_{S}, Y_{S}\right)}$ and $Q_{\left(X_{Q}, Y_{Q}\right)}$ area mixed node, it's necessary to join two pure node from $\left(S_{1}, Q_{1}\right)$ and $\left(S_{2}, Q_{2}\right)$ to calculateB $L(S, Q)$. Then the set of nodes joining $S$ and $Q$ is the union of determined nodes between $\left(S_{1}, Q_{1}\right)$ and $\left(S_{2}, Q_{2}\right)$, and the nodes $S$ and $Q$ as shown in figure 5 .

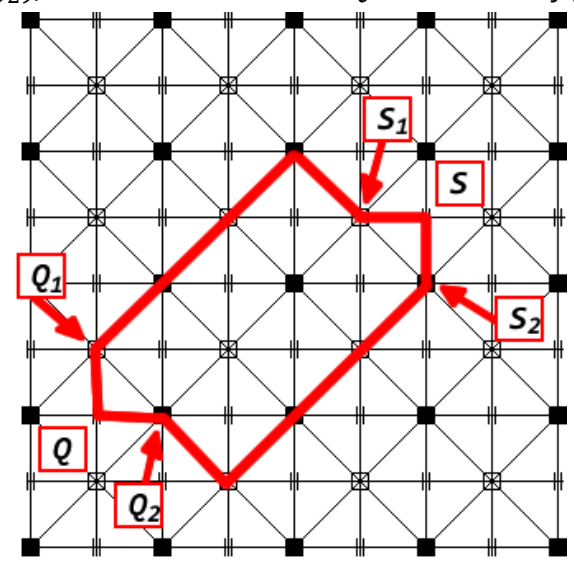

Figure 5: $B L(S, Q)$ when $S$ and $Q$ are mixed nodes

0 Case 3: If $S$ and $Q$ are pure nodes and $\left|X_{S}-X_{Q}\right| \neq\left|Y_{S}-Y_{Q}\right|$, then there is two cases:

a. If $\left|X_{S}-X_{Q}\right|>\left|Y_{S}-Y_{Q}\right|$ then

$$
\mathrm{P} \in \mathrm{BL}(\mathrm{S}, \mathrm{Q}) \Leftrightarrow\left\{\begin{array}{l}
\left|\mathrm{X}_{\mathrm{P}}-\mathrm{X}_{\mathrm{S}}\right|+\left|\mathrm{X}_{\mathrm{Q}}-\mathrm{X}_{\mathrm{p}}\right|=\left|\mathrm{X}_{\mathrm{Q}}-\mathrm{X}_{\mathrm{S}}\right| \\
\left|\mathrm{Y}_{\mathrm{P}}-\mathrm{Y}_{\mathrm{S}}\right| \leq\left|\mathrm{X}_{\mathrm{P}}-\mathrm{X}_{\mathrm{S}}\right| \\
\left|\mathrm{Y}_{\mathrm{Q}}-\mathrm{Y}_{\mathrm{p}}\right| \leq\left|\mathrm{X}_{\mathrm{P}}-\mathrm{X}_{\mathrm{Q}}\right|
\end{array}\right.
$$

In this case, the nodes joining $S$ and $\mathrm{Q}$ are in zone I or III as shown in figure 4.

b. If $\left|X_{S}-X_{Q}\right|<\left|Y_{S}-Y_{Q}\right|$ then

$$
\mathrm{P} \in \mathrm{BL}(\mathrm{S}, \mathrm{Q}) \Leftrightarrow\left\{\begin{array}{l}
\left|\mathrm{Y}_{\mathrm{P}}-\mathrm{Y}_{\mathrm{S}}\right|+\left|\mathrm{Y}_{\mathrm{Q}}-\mathrm{Y}_{\mathrm{p}}\right|=\left|\mathrm{Y}_{\mathrm{Q}}-\mathrm{Y}_{\mathrm{S}}\right| \\
\left|\mathrm{X}_{\mathrm{P}}-\mathrm{X}_{\mathrm{S}}\right| \leq\left|\mathrm{Y}_{\mathrm{P}}-\mathrm{Y}_{\mathrm{S}}\right| \\
\left|\mathrm{X}_{\mathrm{Q}}-\mathrm{X}_{\mathrm{p}}\right| \leq\left|\mathrm{Y}_{\mathrm{P}}-\mathrm{Y}_{\mathrm{Q}}\right|
\end{array}\right.
$$

In this case, the nodes joining $S$ and $\mathrm{Q}$ are in zone II or IV as shown in figure 4.

O Case 4 : If $S$ and $Q$ are not both pure nodes or mixed nodes and $\left|X_{S}-X_{Q}\right| \neq\left|Y_{S}-Y_{Q}\right|$, then there is two cases:

a. $S$ is a pure node and $Q$ is a mixed node and $\left|X_{S}-X_{Q}\right|>\left|Y_{S}-Y_{Q}\right|$, then

$$
\mathrm{P} \in \mathrm{BL}(\mathrm{S}, \mathrm{Q}) \Leftrightarrow \mathrm{P} \in \mathrm{BL}\left(\mathrm{S}, \mathrm{Q}^{\prime}\right) \cup\{\mathrm{Q}\}
$$

with

$$
\mathrm{Q}^{\prime}\left(\mathrm{X}_{\mathrm{Q}}-\frac{\mathrm{X}_{\mathrm{Q}}-\mathrm{X}_{\mathrm{S}}}{\left|\mathrm{X}_{\mathrm{Q}}-\mathrm{X}_{\mathrm{S}}\right|}, \mathrm{Y}_{\mathrm{Q}}\right) \text { a pure node as shown in figure } 6 \text {. }
$$

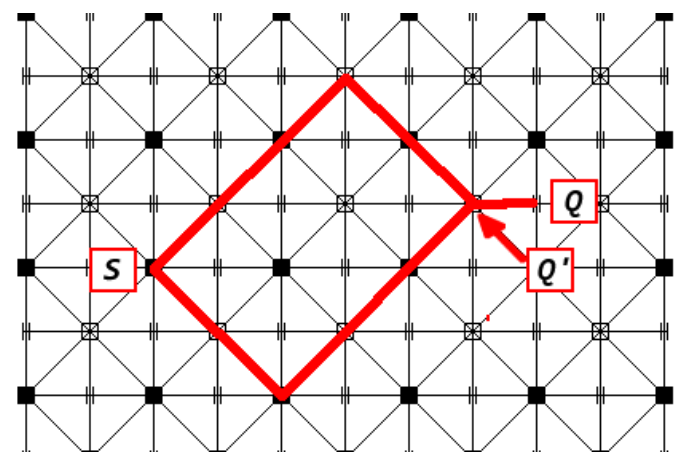

Figure 6: $\mathrm{BL}(\mathrm{S}, \mathrm{Q})$ when $\mathrm{S}$ is a pure node and $\mathrm{Q}$ is a mixed node and $\left|X_{S}-X_{Q}\right|>\left|Y_{S}-Y_{Q}\right|$

b. $S$ is a pure node and $Q$ is a mixed node and $\left|X_{S}-X_{Q}\right|<\left|Y_{S}-Y_{Q}\right|$, then

$$
\mathrm{P} \in \mathrm{BL}(\mathrm{S}, \mathrm{Q}) \Leftrightarrow \mathrm{P} \in \mathrm{BL}\left(\mathrm{S}, \mathrm{Q}^{\prime}\right) \cup\{\mathrm{Q}\}
$$


With $\mathrm{Q}^{\prime}\left(\mathrm{X}_{\mathrm{Q}}, \mathrm{Y}_{\mathrm{Q}}-\frac{\mathrm{Y}_{\mathrm{Q}}-\mathrm{Y}_{\mathrm{S}}}{\left|\mathrm{Y}_{\mathrm{S}}-\mathrm{Y}_{\mathrm{Q}}\right|}\right)$ a pure node as shown in figure 7 .

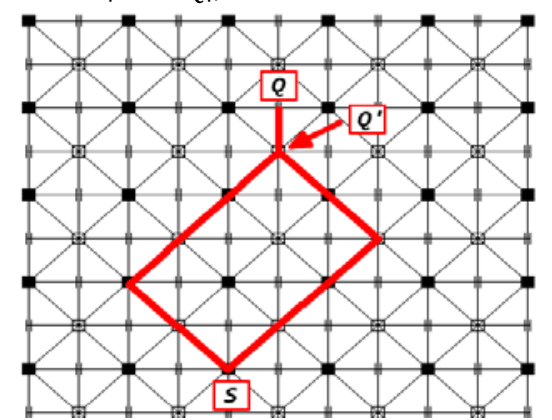

Figure 7: $\mathrm{BL}(\mathrm{S}, \mathrm{Q})$ when $S$ is a pure node and $\mathrm{Q}$ is a mixed node and $\left|X_{S}-X_{Q}\right|<\left|Y_{S}-Y_{Q}\right|$

o Case 5: If $S$ and $Q$ are both mixed nodes and $\left|X_{S}-X_{Q}\right| \neq\left|Y_{S}-Y_{Q}\right|$, then there is two cases:

a. If $\left|X_{S}-X_{Q}\right|>\left|Y_{S}-Y_{Q}\right|$, then

$$
\mathrm{P} \in \mathrm{BL}(\mathrm{S}, \mathrm{Q}) \Leftrightarrow \mathrm{P} \in \mathrm{BL}\left(\mathrm{S}^{\prime}, \mathrm{Q}^{\prime}\right) \cup\{\mathrm{S}, \mathrm{Q}\}
$$

With $\quad S^{\prime}\left(X_{S}-\frac{X_{S}-X_{Q}}{\left|X_{S}-X_{Q}\right|}, Y_{S}\right)$ and $Q^{\prime}\left(X_{Q}-\frac{x_{Q}-X_{S}}{\left|X_{S}-X_{Q}\right|}, Y_{Q}\right)$ are pure nodes as shown in figure 8.

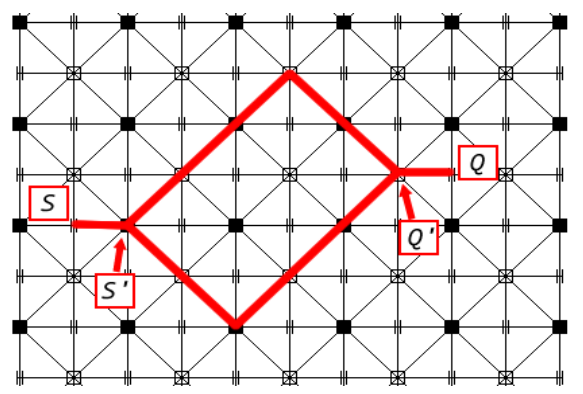

Figure 8: $\mathrm{BL}(\mathrm{S}, \mathrm{Q})$ when $S$ and $\mathrm{Q}$ are both mixed nodes and $\left|X_{S}-X_{Q}\right|>\left|Y_{S}-Y_{Q}\right|$

b. If $\left|X_{S}-X_{Q}\right|<\left|Y_{S}-Y_{Q}\right|$, then

$$
\mathrm{P} \in \mathrm{BL}(\mathrm{S}, \mathrm{Q}) \Leftrightarrow \mathrm{P} \in \mathrm{BL}\left(\mathrm{S}^{\prime}, \mathrm{Q}^{\prime}\right) \cup\{\mathrm{S}, \mathrm{Q}\}
$$

With $S^{\prime}\left(X_{S}, Y_{S}-\frac{Y_{Q}-Y_{S}}{\left|Y_{S}-Y_{Q}\right|}\right)$ and $Q^{\prime}\left(X_{Q}, Y_{Q}-\frac{Y_{Q}-Y_{S}}{\left|Y_{S}-Y_{Q}\right|}\right)$ are pure nodes as shown in figure 9.

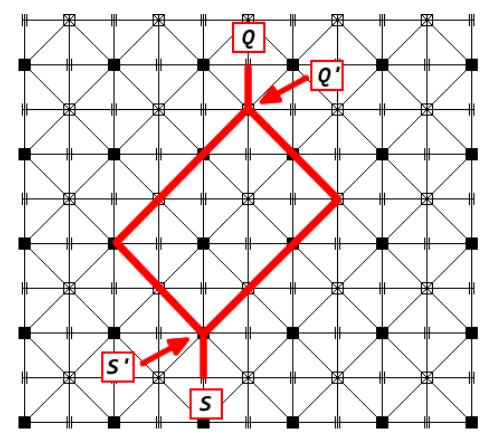

Figure 8: $\mathrm{BL}(\mathrm{S}, \mathrm{Q})$ when $S$ and $\mathrm{Q}$ are both mixed nodes and $\left|X_{S}-X_{Q}\right|<\left|Y_{S}-Y_{Q}\right|$

\subsection{Khalimsky flooding algorithm}

The Khalimsky flooding algorithm determines the relay nodes progressively for transmitting data packets from a source gateway node $Q_{\left(X_{Q}, Y_{Q}\right)}$ to the sink node $S_{(0,0)}$ in BL(S, $\left.Q\right)$. Each node receiving a package, diffuses it once towards the nodes which it has calculated their coordinates. For 
each node in $\mathrm{BL}(\mathrm{S}, \mathrm{Q})$, there is one, two or three gateway nodes to forward data in each step according to the Khalimsky theory as shown in the figure 9.

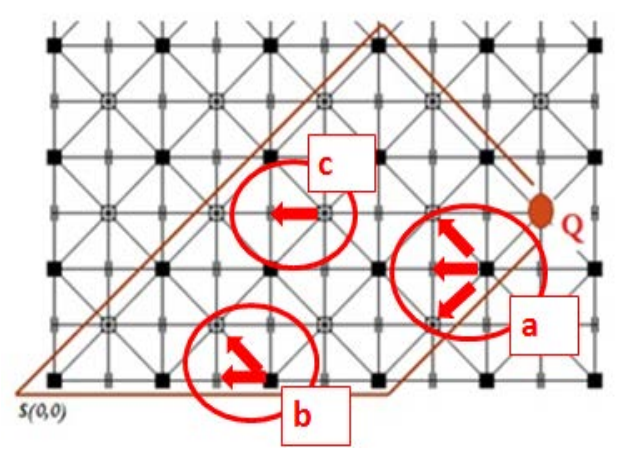

Figure 9: Khalimsky flooding cases of forwarding packets in $\mathrm{BL}(S, Q)$; (a): three gateway nodes;

(b): two gateway nodes; (c): one gateway node

According to the five cases of the Khalimsky theory, in the previous subsection, we have developed the following flooding algorithm which permits to calculate the $\mathrm{BL}(\mathrm{S}, \mathrm{Q})$ and the gateway nodes, hope by hope, in the process of data transmission.

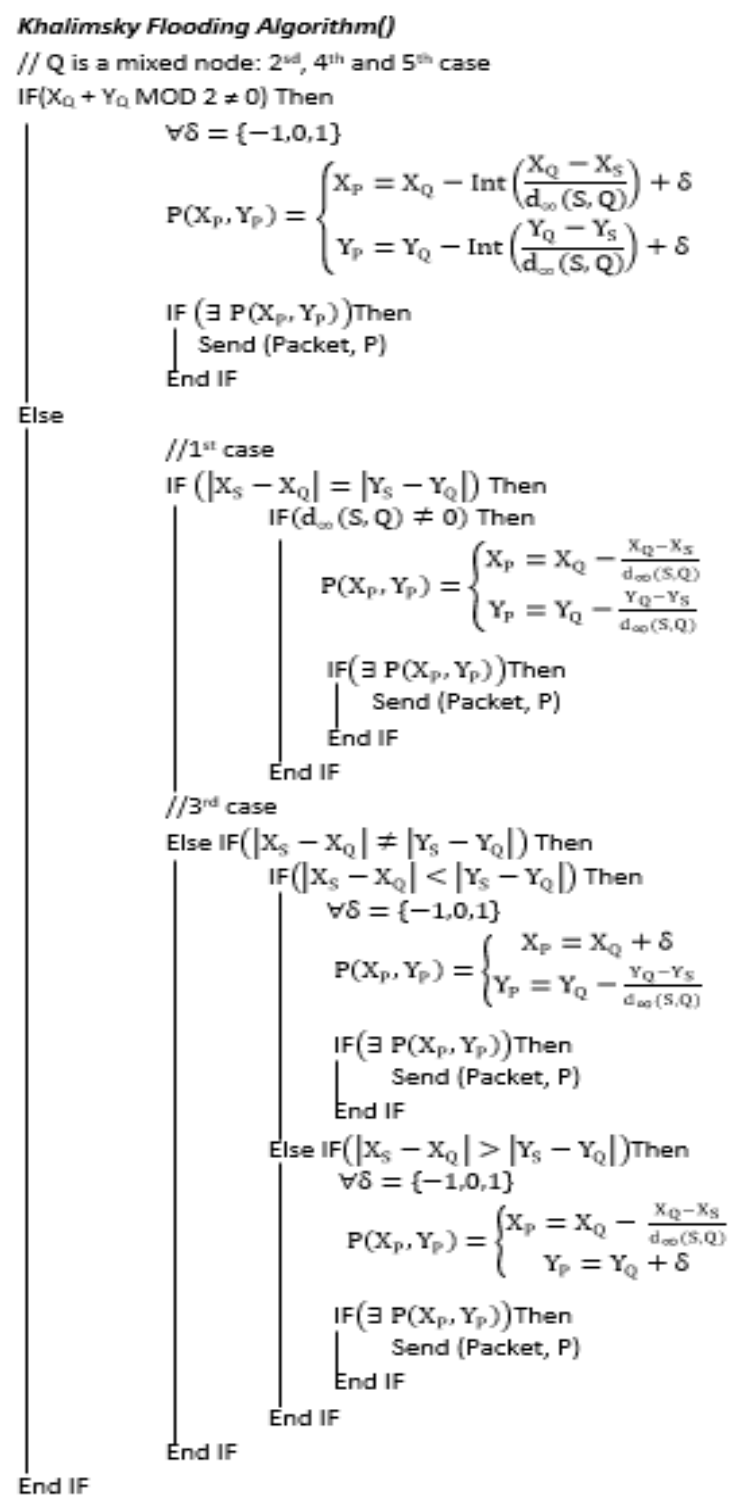

Algorithm 2: Khalimsky flooding algorithm 


\section{Simulation results}

To evaluate the performance of the Khalimsky theory applied on the WSNs area, we used the TOSSIM simulator. We simulated a WSN composed of 50 MicaZ sensors where the energy consumption is modeled by the PowerTOSSIM-Z plugin, compatible with TinyOS2.1, for each node [5-7]. The sensors are deployed hierarchically and uniformly according to the Khalimsky architecture in a sector of the plane, with a sink placed at the center of the sector as shown in figure 2 . This topology is defined in a specific file that we called "topology.txt" in TOSSIM to configure the links and adjacency lists between the simulated sensors as shown in figure 10. This file defines the gain in the $4^{\text {th }}$ column between the source node in the $2^{\text {ed }}$ column and the destination node in the $3^{\text {rd }}$ column.

\begin{tabular}{|llll|}
\hline $\operatorname{gain}$ & 0 & 1 & -54 \\
$\operatorname{gain}$ & 0 & 2 & -72 \\
$\operatorname{gain}$ & 0 & 3 & -54 \\
$\operatorname{gain}$ & 1 & 0 & -54 \\
$\operatorname{gain}$ & 1 & 2 & -54 \\
$\operatorname{gain}$ & 1 & 4 & -54 \\
$\operatorname{gain}$ & 2 & 0 & -72 \\
$\operatorname{gain}$ & 2 & 1 & -54 \\
$\operatorname{gain}$ & 2 & 3 & -54 \\
$\operatorname{gain} 2$ & 4 & -72 \\
$\operatorname{gain}$ & 2 & 5 & -54 \\
$\operatorname{gain} 2$ & 6 & -72 \\
$\operatorname{gain} 2$ & 7 & -54 \\
$\operatorname{gain}$ & 2 & 8 & -72 \\
$\operatorname{la}$ & & & \\
\hline
\end{tabular}

Figure 10: extract of topology file configuration in TOSSIM simulator

To launch the simulation we have developed a program file written in the Python language. This program is used to load entities, representing the sensors in the computer memory as the Khalimsky topology file previously created, to apply noise model and to boot sensors starting with the Sink. Figure 11 shows an extract of the Python program that we used to run the simulation. This extract contains three parts. The first part allows the loading of the topology file previously created. The second part concerns the application of the noise model and the start sensor nodes. The third section determines the time of simulation and run each program of sensors.

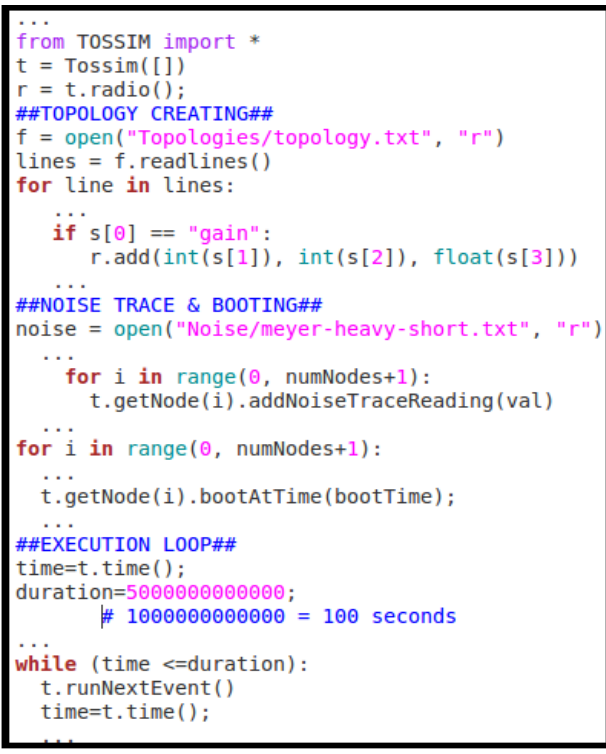

Figure 11: Extract of Python program running the simulation

When TOSSIM run simulation, each booted sensor node run the auto-organization algorithm that we developed in algorithm 1 to calculate its coordinates. Figure 12 shows a part of the result of the 
execution of this algorithm for several sensors. It shows the sensor ID in TOSSIM, the calculated coordinates and the moment of reaching.

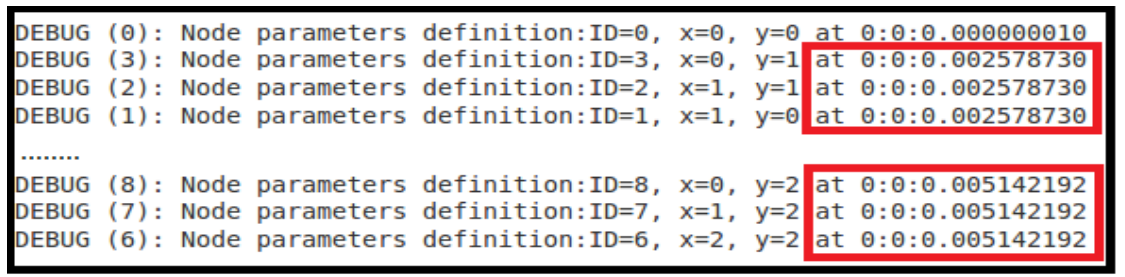

Figure 12: Auto-organization algorithm results

The last part of the Python program consists to run the program of each simulated sensor according to the Khalimsky theory. Each sensor has a timer that organizes these tasks. These tasks are called evenements. The main activities of a sensor are the data collecting from its environment and the routing of created and received packets. Figure 13 shows a part of the execution result of the routed packet by Khalimsky flooding algorithm from the node 31 via the node 20 to the Sink. The node 20, after calculating the destination node selects the node 12 to pass the received packet. It is the case 1 in Khalimsky flooding algorithm since the node 20 is a pure node and its coordinates are $x=y=4$.

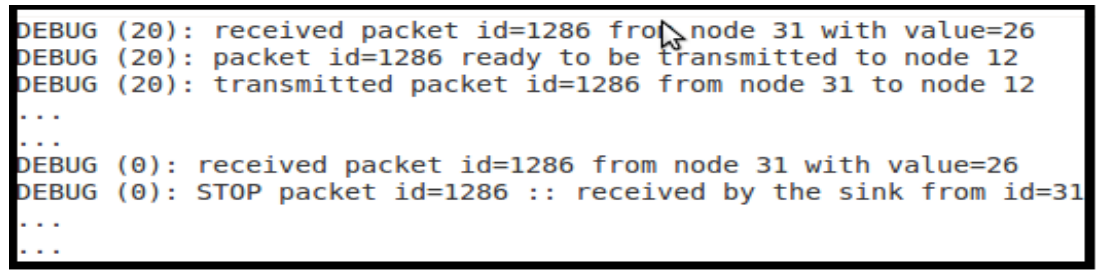

Figure 13: Result part of Khalimsky flooding algorithm simulation

Well TOSSIM simulator that can simulate a WSN, it is not possible to model the energy consumption. PowerTOSSIM-Z is a plugin compatible with TinyOS2.1 used to model the energy consumption of each sensor. It uses the results provided by TOSSIM to determine the energy consumed by the sensor nodes. Figure 14 shows the total consumed energy by the node 36 and the simulated time. It shows also the energy consumed by the different components in the node when it's possible to ignore among them. All nodes have the same power $21600000 \mathrm{~mJ}$ when starting the simulation.

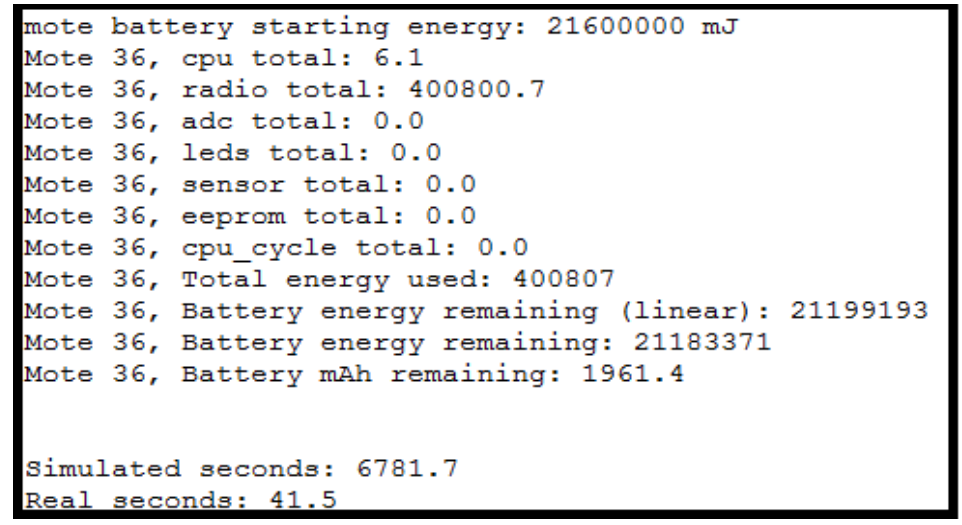

Figure 14: PowerTOSSIM-Z energy modeling results part

In the following, our evaluation is based on comparing the flooding rates and the energy consumption of conventional flooding algorithm versus Khalimsky flooding algorithm by the analysis of result files generated by TOSSIM simulator and PowerTOSSIM-Z as shown in Figure 14. 
Figure 15 shows the flooding rates values of conventional flooding algorithm versus Khalimsky flooding algorithm. At the beginning the two algorithms have the same values of the flooding rates. After 11 hours, the conventional flooding algorithm attains 13242250 transmissions while the Khalimsky flooding algorithm makes 9189662 transmissions. After 23 hours, the conventional flooding algorithm attains 25098762 transmissions while the Khalimsky flooding algorithm makes 18434604 transmissions.

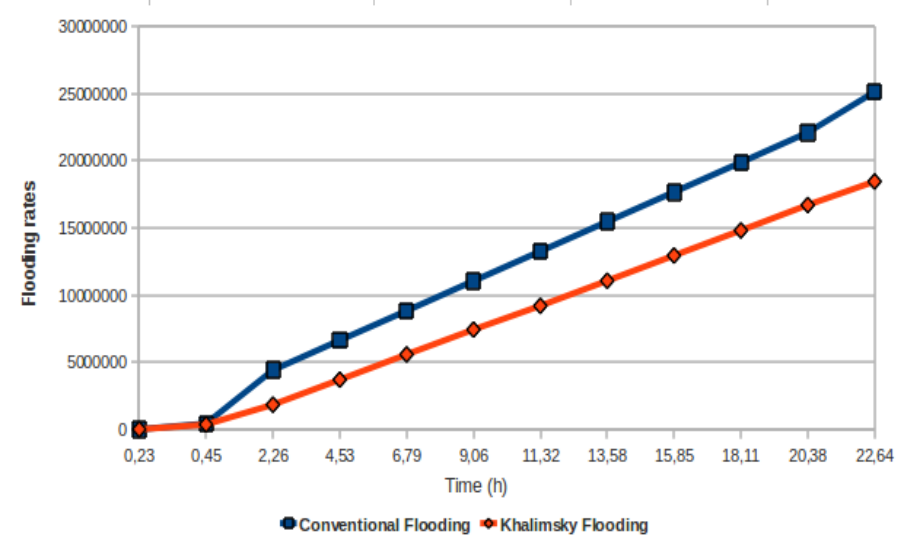

Figure 15: Flooding rates

According to the obtained results, we denote that the Khalimsky topology allowed reducing the flooding rates to about $31 \%$.

Figure 16 shows the energy consumption difference between conventional flooding algorithm and Khalimsky flooding algorithm. The amount of dissipated energy applying the Khalimsky theory is very lower than the second algorithm. At the beginning all nodes have $21600000 \mathrm{~mJ}$. After 11 hours, in the conventional flooding algorithm the consumed energy attain $10000000 \mathrm{~mJ}$ while in the Khalimsky flooding algorithm the value is $4000000 \mathrm{~mJ}$. After 23 hours, the conventional flooding algorithm makes $21000000 \mathrm{~mJ}$ when the Khalimsky flooding algorithm does not exceed $6000000 \mathrm{~mJ}$.

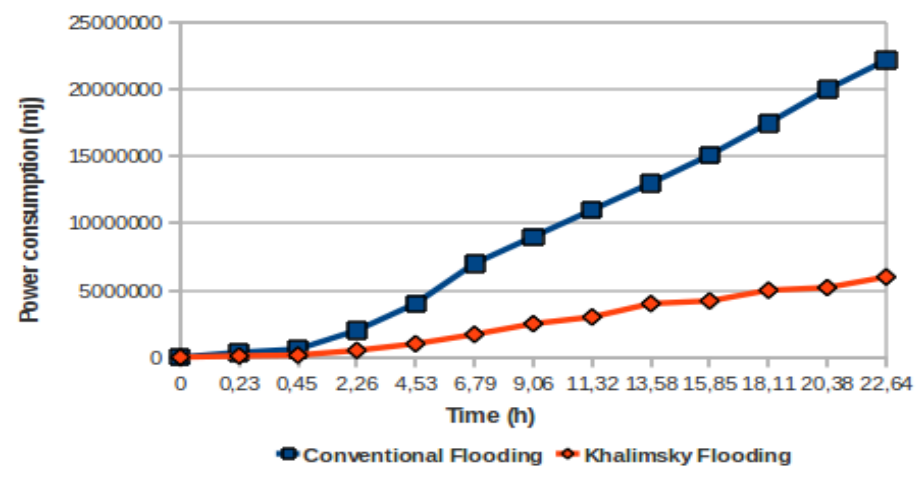

Figure 16: Flooding power consumption results

According to the power consumption obtained results, we denote that the energy consumption average gain obtained after 11 hours is about $50 \%$ but after 23 hours is about $75 \%$.

We deduct from the two figures figure 15 and figure 16 an important gap between the red and the blue curves (representing the energy consumption and the flooding rates of Khalimsky flooding algorithm and the conventional flooding approach). The Khalimsky topology reduces the links 
between sensors. When a node sends a data packet by flooding, the number of used sensors determined by Khalimsky theory is limited by nodes forming optimal paths as shown in figure 3 .

\section{Advantages \& Future Works}

Our solution for deploying a WSN based on the topology of Khalimsky can be applied effectively in many areas such as domotic field and industrial field, for monitoring and building security of infrastructures and industrial areas against fires, gas leakage and pollution. In these areas, it is necessary to deploy fixed nodes acting as gateways to convey the data routing toward base station. These gateways provide the transfer of data collected by sensors members grouped into clusters to the base station. The collected data can be important and have a real-time aspect. In this case, the data must be transmitted to the base station in a safe and immediately manner. So with our flooding solution, based on the Khalimsky theory, it's possible to perform the tasks in an optimal way with minimal data redundancy and the minimum consumed energy.

For future works, we have used the Khalimsky theory to develop a routing protocol for WSNs, based on the clustering method and the multi-agent systems. These WSNs may contain sensors randomly deployed and prefixed sensors acting as gateway. The randomly deployed sensors are grouped into clusters. Each cluster must be able to reach one or more gateways. Gateway sensors are deployed according to the Khalimsky topology. The simulation tests, in the process, showed encouraging results.

\section{Conclusion}

In this paper, we have been developed a novel hierarchical topology for WSNs based on mathematical Khalimsky theory. This method calculates a subset of nodes to use as gateways for rooting data between a source node and a destination node. This subset of these nodes includes all optimal paths. However, a diffused data packet by a node will never go through outside this subset. This implies the reduction of the flooding rates in a WSN. Thereafter, a considerable minimization of the energy consumption is denoted. This method was validated by a simulation of a WSN using TOSSIM and PowerTossim-Z in TinyOS2.1 that allows the energy consumption modeling. The Khalimsky protocol has a great capacity of energy conservation and of flooding rates reduction. Following these simulations, we clearly note the optimality and the effectiveness of the Khalimsky flooding algorithm compared to the conventional flooding algorithm in term of energy consumption and flooding rates.

\section{REFERENCES}

[1]. M. Mezghani, G. Ellouze, A. Grati, I. Bouabidi, M. Abdellaoui, Multitasks-Generic platform via WSN. International Journal of Distributed and Parallel Systems (IJDPS), Vol.2, No.4, July 2011, p. 54-67.

[2]. A. Nayak, I. Stojmenovic,Wireless Sensor and Actuator Networks: algorithms and protocols for scalable coordination and data communication. WILEY series, 2010, chapter 4 and 5, p. 95-152.

[3]. G. Anastasi, M. Conti, M. Di Francesco, A. Passarella,Energy conservation in wireless sensor networks: A survey, Ad Hoc Networks,7 (2009), p. 537-568.

[4]. Y. Youssef, Routage pour la gestion de l'energie dans les réseaux de capteurs sans fil. These de doctorat, Haute Alsace-France, Juillet 2010. 
[5]. M. Mezghani, O. Mezghani, H. Rekik, M. Abdellaoui, TinyOS2.1 with nesC, TOSSIM and PowerTOSSIM-Z Emulation and Simulation Environments to Networked Domotic Embedded Systems.12th International conference on Sciences and Techniques of Automatic control and computer engineering (STA2011), December 18-20, 2011, Sousse, Tunisia.

[6]. E. Perla , A. O. Cathain, R. S. Carbajo, PowerTOSSIM-z: Realistic Energy Modelling for Wireless Sensor Network Environments. Proceedings of the 3nd ACM workshop on Performance monitoring and measurement of heterogeneous wireless and wired networks, October 31, 2008, Vancouver, BC, Canada, p. 35-42.

[7]. P. Levis, D. Gay, TinyOS programming. Cambridge University Press, 2009, p. 3-105.

[8]. M. Hadjila, M. Fehman, A comparative study of the wireless sensor networks routing protocols scalability. International Journal of Distributed and Parallel Systems (IJDPS), Vol.2, No.4, July 2011, p. 26-33.

[9]. K. Beydoun, Conception d'un protocole de routage hiérarchique pour les réseaux de capteurs. Thèse de doctorat, Franche-Comte-France, Décembre 2012.

[10]. M. Lehsaini, Diffusion et couverture basées sur le clustering dans les réseaux de capteurs: aplication à la domotique. Thèse de doctorat, Franche-Comte-France, Juillet 2009.

[11]. E. Bouacida, The jordan curve theorem in the Khalimsky plane, Applied General Topology 9, 252 (2008).

[12]. U. Eckhardt, L.J. Latecki, Topologies for the digital spaces Z2 and Z3. Comput. Vision Image Understanding, 90 (2003), p. 295-312.

[13]. E.D. Khalimsky, On topologies of generalized segments. Soviet Math. Dokl., 10 (1999), p. 15081511.

[14]. E.D. Khalimsky, R. Kopperman, P.R. Meyer, Boundaries in digital planes. J. Appl. Math. Stoch. Anal. 3 (1990), p. 27-55.

[15]. E. Khalimsky, R. Kopperman, P. R. Meyer, Computer graphics and connected topologies on finite ordered sets.Topol. Appl. 36, 1 (1990). 